\title{
Multivariate Method Based on Raman Spectroscopy for Quantification of Dipyrone in Oral Solutions
}

\author{
Luciana Lopes Guimarães $\mathbb{D}^{1,2}$ Letícia Parada Moreira, ${ }^{1,2}$ Bárbara Faria Lourenço, \\ Walber Toma, ${ }^{1,2}$ Renato Amaro Zângaro, ${ }^{3}$ Marcos Tadeu Tavares Pacheco, ${ }^{2,3}$ \\ and Landulfo Silveira Jr. $\mathbb{D}^{2,3}$ \\ ${ }^{1}$ Laboratory of Natural Products, Department of Pharmaceutical Sciences, Universidade Santa Cecília (UNISANTA), Rua Cesário \\ Mota, 8, F 83A, 11045-040 Santos, SP, Brazil \\ ${ }^{2}$ Department of Ecology, Universidade Santa Cecília (UNISANTA), Rua Cesário Mota, 8, 11045-040 Santos, SP, Brazil \\ ${ }^{3}$ Center for Innovation, Technology and Education-CITE, Universidade Anhembi Morumbi (UAM), Estr. Dr. Altino Bondensan, 500, \\ 12247-016 São José dos Campos, SP, Brazil
}

Correspondence should be addressed to Luciana Lopes Guimarães; lubioq@gmail.com

Received 6 December 2017; Revised 1 March 2018; Accepted 19 March 2018; Published 23 April 2018

Academic Editor: Simona C. Pinzaru

Copyright () 2018 Luciana Lopes Guimarães et al. This is an open access article distributed under the Creative Commons Attribution License, which permits unrestricted use, distribution, and reproduction in any medium, provided the original work is properly cited.

\begin{abstract}
This work employed a quantitative model based on Raman spectroscopy and principal component regression (RS/PCR) to quantify the active ingredient dipyrone (metamizole) in commercially available formulations as an analytical methodology for quality control in the pharmaceutical industry. Raman spectra were collected using a dispersive Raman spectrometer $(830 \mathrm{~nm}, 250 \mathrm{~mW}$ excitation, and $20 \mathrm{~s}$ exposure time) coupled to a Raman probe. Solutions of dipyrone diluted in water in the range of 80 to $120 \%$ of the concentration of commercial formulations $(500 \mathrm{mg} / \mathrm{mL})$ were used to develop a calibration model based on PCR to obtain the figures of merit for class I validation from the Brazilian Sanitary Surveillance Agency (ANVISA, RE no. 899/2003). This spectral model was then used to predict the concentration of dipyrone in commercial formulations from distinct brands with $500 \mathrm{mg} / \mathrm{mL}$. A prediction error of $6.5 \mathrm{mg} / \mathrm{mL}$ (1.3\%) was found for this PCR model using the diluted samples. Commercial formulations had predicted concentrations with a difference below 5.0\% compared to the label concentration, indicating the applicability of Raman spectroscopy for quality control in the final product.
\end{abstract}

\section{Introduction}

The dipyrone metamizole is a popular analgesic, nonopioid drug, which is commonly used in human and veterinary medicine for pain management in patients where nonsteroidal anti-inflammatory drugs (NSAIDs) are contraindicated [1]. Apart from its analgesic effect, the medication is an antipyretic and spasmolytic agent. Metamizole is predominantly applied in the therapy of pain of different etiologies, of spastic conditions, especially affecting the digestive tract, and of fever refractory to other treatments. It is a relatively safe pharmaceutical preparation, although it is not completely free from undesirable effects. Among these side effects, the most serious one that raises the most controversy is the myelotoxic effect. Despite the evidence showing no risk of teratogenic and embryotoxic effects, the drug must not be administered to pregnant women [2].

Quality control is responsible for verifying that a pharmaceutical product complies with the specifications described in the pharmacopoeias [3]. Routine techniques for measuring dipyrone in industry's quality control include titration in oral metamizole solutions [4]. The process analytical technology (PAT) aims at the development of strategies to the primary and secondary control of the production processes in the pharmaceutical industry. By definition of the Food and Drug Administration (FDA), PAT includes systems for the analysis and control of production processes, based on opportune measures of the quality parameters and 
critical attributes of the material performance, optimizing the production time, energy, and material, thus contributing to sustainability and reducing environmental impact [5]. The PAT Guide announcement by FDA in 2004 was aimed at research into new and faster analytical methodologies to be used on-line and at-line. Optical techniques such as absorption spectroscopy and Raman spectroscopy have been claimed to be consistent, reliable, rapid, and low-cost analyses of chemical species in the industrial field for analytical instrumentation [5-7], which could successfully meet the PAT requirements.

Due to the increased interest in new equipment and techniques for the quality control of drugs, the Brazilian Sanitary Surveillance Agency (ANVISA) created resolutions to regulate such procedures and equipment to be considered during the validation of analytical methods, referring to the qualitative, semiquantitative and/or quantitative validation of drugs and other substances in pharmaceutical products. The Brazilian resolution RE no. 899/2003 states that the validation should guarantee, through experimental studies, that the method meets the requirements of analytical applications, ensuring the reliability of results [8]. It should provide specificity, linearity, range, precision (repeatability), sensitivity, robustness, accuracy, and limit of detection and quantification appropriate to the analysis. RE 899/2003, which is mostly based on the guidelines developed by the ICH Expert Working Group under the International Conference on Harmonisation of Technical Requirements for Registration of Pharmaceuticals for Human Use [9, 10], classifies the procedures into four distinct classes according to their purpose; for each class, a set of tests is required. The validation method for the quantification of commercial pharmaceutical drugs can be accomplished by class I, in which the figures of merit required are specificity, linearity, range, precision, robustness, and accuracy [8].

Classical techniques for the evaluation of pharmaceutical drugs are based on liquid chromatography followed by mass spectrometry (LC/MS). Although sensitive and very specific, LC/MS techniques are time-consuming and present excessive costs, requiring sample preprocessing prior to analysis, which may destroy the sample. To address this problem, optical techniques have been proposed for the quantitative determination of active pharmaceutical ingredients (API) in drugs and medicines, most of which are based on absorption and reflectance spectrometric techniques in the ultravioletvisible and infrared regions [11-13], with advantages such as rapid API evaluation with minimal or even no sample preparation [11].

Vibrational spectroscopy, particularly Raman spectroscopy, has been used in the quantitative analysis of API, with advantages such as the need for small amounts of sample, no sample preparation, no sample destruction, and a very short time required to perform the analysis [11]. Raman spectroscopy can be applied for qualitative and quantitative drug analysis, being one of the techniques of greatest interest in pharmaceutical applications [14]. Raman spectra can be obtained from solids, liquids, and gases, either directly, in glass containers or tubes or even through blisters [15]. In this sense, several pharmaceutical presentations have been evaluated by Raman spectroscopy, such as pills, tablets, capsules, solutions, suspensions, and injectable solutions, among others, aiming at the quantification of the API and other formulation constituents [15-17]. Raman spectral information provides information about the sample's chemical structure, molecular composition of the study material, and quantification $[16,18]$. Dispersive Raman spectrometers make fast scanning/imaging possible using an imaging spectrograph and multichannel detector such as a Peltier-cooled charge-coupled device (CCD) camera [18]; the use of near-infrared excitation (830 and $1064 \mathrm{~nm}$ wavelength) would diminish or eliminate the sample's intrinsic fluorescence $[18,19]$.

The prediction of the amount of a specific compound in a sample using the spectral information can be reached with suitable chemometric techniques [20]. These techniques have a great impact in the pharmaceutical area, since it deals with the analysis of chemical data of multivariate nature, processed in multichannel equipment, where the intensities of the absorption, scattering, or emission in different wavelengths are recorded in a single spectrum [21]. Chemometrics uses mathematical and statistical methods to design experiments in an optimized way and to provide as much chemical information as possible from the obtained data [20-22].

Chemometric tools commonly used in analytical chemistry include partial least squares regression (PLSR) [23] and principal component regression (PCR) [24]. Both techniques are efficient quantitative methods for dealing with experimental noise, collinearities, and nonlinearities and are sufficiently robust to allow the inclusion of new samples to the model with minimum or no changes in the input parameters. Another advantage of the multivariate methods is the possibility to develop a multivariate calibration model, even with the presence of interferences, whether these are of known or unknown nature [21]. PLSR and PCR are methods intended to model response variables (concentrations) when there are a large number of predictor variables (spectra), and those predictors are highly correlated or even collinear. These methods extract new predictor variables from the dataset, known as components, as linear combinations of the original predictor variables, but each technique extracts those components differently. PCR extracts the components with high variations in the predictor variables, without considering the response variable (concentration), whereas PLSR creates these components taking the response variable into account and therefore can model "complicated" data and is often able to fit the response variable with fewer components [25]. PCR uses the principal component scores (the PCs), which are calculated by applying principal component analysis (PCA) in the dataset composed of spectra taken from the samples in known concentrations, and uses these scores (which can be correlated to the concentrations) and the concentrations to develop a linear regression model to estimate the samples' concentration [23-25]. The number of scores that will be used in the linear model depends on the amount of variation presented in the data set, which is determined by the spectral features present in the principal component loading vectors. The first principal component vectors describe the directions 
of the maximum variance and are normally related to the spectral features of the API in the drug formulation; therefore, these PCs are normally used in the model.

Optical spectroscopy and multivariate calibration, mainly PLSR and PCR, have been applied in the pharmaceutical field to calculate the amount of API in prepared solutions or directly in the commercial medicines, such as quantitative determination of acetylsalicylic acid and acetaminophen in tablets by FT-Raman using both PCR and PLSR [26]; identification of caffeine, acetaminophen, and acetylsalicylic acid in a mixed solution using ultraviolet absorption and PLSR [22]; the simultaneous quantification of samples containing mixtures of amiloride hydrochloride, atenolol, hydrochlorothiazide, and timolol maleate using classical least squares, PCR, and PLS applied on UV spectral data, evidencing similar results for the PCR and PLS models [27]; the simultaneous determination of sodium dipyrone and papaverine hydrochloride based on PLSR and ultraviolet spectrophotometric measurements in commercial formulation $\left(\mathrm{Melpaz}^{\circledR}\right)$ [28]; the quantification of diclofenac sodium in tablets and capsules by means of FT-Raman and PLSR, PCR, and counterpropagation artificial neural networks [29]; the quantification of nimesulide in excipient and commercial samples using FT-NIR absorption compared to ultraviolet absorption and the PLSR model [30]; and the novel application of inkjet-printed pharmaceuticals such as haloperidol, used as a model API, and lactic acid and ethanol, used as printable ink bases, applied to three different inorganic and organic substrates and with the quantification of haloperidol performed by the PLS model [31].

Although dipyrone has great relevance in Brazilian clinical practice [32], commercialization of this drug is prohibited in most developed countries in the face of severe adverse reactions that may occur, such as the depressive effect of the bone marrow, which may result in aplastic anemia and, mainly, agranulocytes $[33,34]$. There are few studies in the literature that have addressed the quality control of dipyrone production. Therefore, the purpose of this study was to evaluate the feasibility of Raman spectroscopy to assay the API concentration in water solutions containing dipyrone using a spectral model based on principal component regression (PCR) in attending the figures of merit of the Brazilian Agency ANVISA for class I validation (specificity, linearity, range, precision, robustness, and accuracy), in order to assess the ability of Raman spectra to be used as an analytical technique according to the ANVISA and PAT requirements. The developed quantitative model was then employed to predict the concentration of dipyrone in samples of commercial formulations (drop's presentation) containing this API with $500 \mathrm{mg} / \mathrm{mL}$, aiming its use as an analytical technique of choice for quantitative validation.

\section{Materials and Methods}

2.1. Solution Preparation. Solutions with seven different concentrations of dipyrone were prepared in distilled water $(400,450,475,500,525,550$, and $600 \mathrm{mg} / \mathrm{mL})$, representing the range from 80 to $120 \%$ of the concentration found in the commercially available drug containing dipyrone. These samples were prepared in triplicate. Also, 10 flasks of medicines in drops containing dipyrone with $500 \mathrm{mg} / \mathrm{mL}$ of API produced by several laboratories (4 generics, 4 similar, and 2 reference brands) were obtained from a local drugstore.

2.2. Raman Spectroscopy. The dipyrone-prepared samples and commercial medicines were submitted to Raman spectroscopy using a benchtop Raman spectrometer (model Dimension P-1, Lambda Solutions Inc., Waltham, MA, USA). The laser is coupled to a fiber-optic-based Raman probe (model Vector Probe, Lambda Solutions Inc., Waltham, MA, USA), as shown in Figure 1. The spectrometer is composed of a diode laser $(830 \mathrm{~nm})$, an imaging spectrograph, and a high-sensitivity charge-coupled device (CCD) camera. The system is controlled by a computer. The laser power at the Raman probe tip was adjusted to $250 \mathrm{~mW}$. The dilutions of dipyrone and solutions of commercial medicines were placed in an aluminum sample holder with holes of about $80 \mu \mathrm{L}$ for Raman measurements, and the Raman signal was collected in 10 scans of $2 \mathrm{~s}$ each. All spectra were collected at the same day and experimental conditions in triplicate.

The procedures of spectral calibration and preprocessing (fluorescence background subtraction) were performed using a routine developed under Matlab 7.4 (The MathWorks Inc., Natick, MA, USA). The Raman shift calibration was performed by collecting the spectrum of naphthalene and correlating the spectrum band positions (pixel) with the known band shift $\left(\mathrm{cm}^{-1}\right)$ with a 3rd-order polynomial fitting. The intensity response of all optical components as a function of the wavelength was obtained using a tungsten calibration lamp. To remove the fluorescence background, the polynomial baseline correction proposed by Lieber and Mahadevan-Jansen [35] was performed, in which a 5thorder polynomial is fitted in the minima of the spectra, and this polynomial is subtracted from the gross spectrum, maintaining the high-frequency Raman bands.

2.3. PCR Quantitative Model. In order to develop a model for the quantitative analysis of API (dipyrone) in commercial solutions, a quantitative model using principal component regression (PCR) was developed using the spectra of the API dilutions in the concentration range from 400 to $600 \mathrm{mg} / \mathrm{mL}$ and the nominal concentrations. Then, the linear equation from this model was applied to estimate the concentrations of dipyrone in commercial solutions.

For the PCR model, the principal component analysis (PCA) was initially employed in the spectra of dilutions of $80-120 \%$ of dipyrone in water, in order to decompose the spectra onto the principal component loading vectors (LVs) and scores (PCs). In the PCA decomposition, the LVs indicate the spectral features that are important in the diluted samples, and the PCs indicate the intensities of these LVs in each spectrum. PCA was calculated using the function princomp. $m$ through the software Matlab 7.4/Statistics Toolbox (The MathWorks Inc., Waltham, MA, USA).

The quantitative PCR model was built using PC1, since it has been demonstrated that LV1 represents $99 \%$ of the 


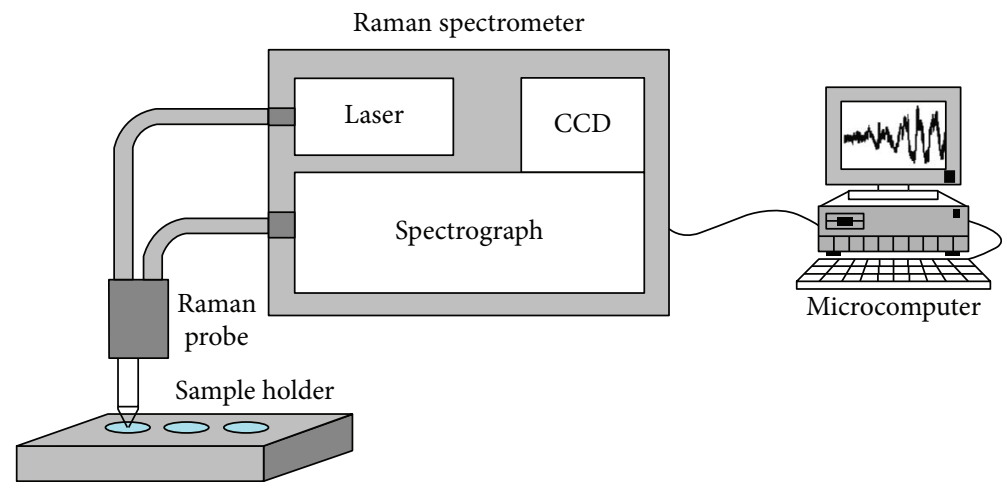

FIGURE 1: Schematic diagram of the dispersive Raman spectrometer used in the experiment. Laser power measured at the Raman probe tip: $250 \mathrm{~mW}$, wavelength: $830 \mathrm{~nm}$, spectrometer resolution in the $400-1800 \mathrm{~cm}^{-1}$ range: $2 \mathrm{~cm}^{-1}$.

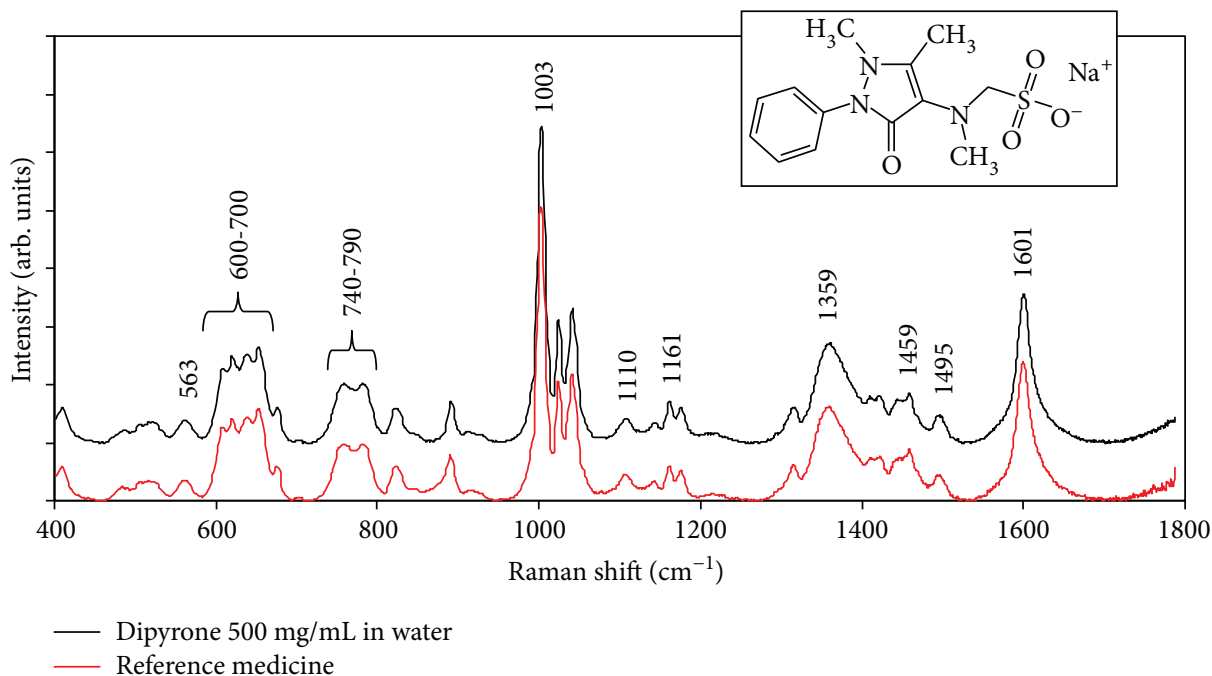

Figure 2: Plot of the spectrum of dipyrone diluted in water in the concentration of $500 \mathrm{mg} / \mathrm{mL}(100 \%$ dilution) and the first three principal component loading vectors (LVs), indicating that LV1 is related to the active ingredient dipyrone. LV2 and LV3 do not carry relevant spectral features.

spectral variation in the diluted sample dataset (plot of the spectral information present in the LV1 is seen in Figure 2, Results). For the development of the model, PC1 of the triplicates was plotted versus the concentration of API in the dilution, and the linear equation (PC ordinate, API concentration abscissa) was obtained. Thus, the concentrations of diluted samples were estimated through this equation. These triplicates were used to obtain the figures of merit according to the ANVISA, class I validation and the IUPAC recommendations [36].

Finally, the linear equation was used to estimate the concentration of API in the solutions of commercial medicines. For this, the first step is to obtain the PCs that are present in each sample spectrum of commercial medicine and needed for the ordinate in the regression equation. The PCs were estimated by least-square fitting the first LVs and the sample spectrum, where the fitting coefficients were referred to these "new PCs" of each LV present in each Raman spectrum of the commercial medicines. The fitting was performed using lsqlin.m under Matlab 7.4/Optimization
Toolbox. PC1 estimated by least-square fitting was used in the linear equation, and the concentration of the API was then obtained.

2.4. Figures of Merit according to ANVISA. The predicted concentrations in the replicate samples were submitted to ANVISA criteria [8], with the figures of merit for class I validation:

(i) Range: in this study, a range of 400 to $600 \mathrm{mg} / \mathrm{mL}$ was determined.

(ii) Specificity/selectivity: in order to verify whether the proposed technique is able to identify and quantify dipyrone, even in different excipients, a solution of dipyrone $(500 \mathrm{mg} / \mathrm{mL})$ in pure water and dipyrone $(500 \mathrm{mg} / \mathrm{mL})$ from the reference commercial medicine (3 replicates of each) was considered.

(iii) Linearity: the curve slope $(m)$, which determines the proportionality of the analytical function, the linear 
coefficient $(h)$, which is used to represent the correlation, and the correlation coefficient $(r)$ were calculated. The criterion for acceptance is $r=0.99$.

(iv) Precision: for the study, the concentrations 400, 500, and $600 \mathrm{mg} / \mathrm{mL}$ were used in triplicate, totaling nine solutions. The precision was expressed as relative standard deviation (RSD), according to

$$
\mathrm{RSD}=\frac{\mathrm{SD}}{\mathrm{DAC}} \times 100,
$$

where $\mathrm{SD}=$ standard deviation and $\mathrm{DAC}=$ determined average concentration of the model's measures. SD is calculated as

$$
\mathrm{SD}=\sqrt{\frac{\sum_{i=1}^{n}(\mathrm{VO}-\mathrm{DAC})^{2}}{n-1}}
$$

where $\mathrm{VO}=$ value obtained in the test, $\mathrm{DAC}=$ determined average concentration, and $n=$ number of samples. For the ANVISA, the maximum RSD for the method should not exceed $5 \%$.

(v) Accuracy (recovery): this was determined from nine determinations in the range of the procedure, that is, concentrations of 400,500 , and $600 \mathrm{mg} / \mathrm{mL}$, in triplicate. The equation used to calculate the accuracy $(\mathrm{AC})$ is

$$
\mathrm{AC}=\frac{\mathrm{DAC}}{\mathrm{VA}} \times 100,
$$

where VA is value added.

\section{Results}

3.1. Raman Spectra of Diluted Solutions and Development of the Spectral Model. The Raman spectrum of dipyrone diluted in water at a concentration of $500 \mathrm{mg} / \mathrm{mL}$ and the spectrum of a reference medicine are shown in Figure 3. Both spectra show the vibrational bands of dipyrone (labeled peaks), and band assignments are shown in Table 1. No observable or relevant excipient peak was observed.

The data set of the diluted samples was submitted to PCA, and the output variables (principal component loading vectors-LVs-and scores-PCs) were obtained. Figure 2 presents the first $3 \mathrm{LVs}$ and the spectra of dipyrone diluted in water, showing that vector 1 is strongly related to the active ingredient dipyrone. Therefore, $\mathrm{PC} 1$ can be used to estimate the concentration of dipyrone in a linear regression model. This model, presented in Figure 4(a), shows the correlation between the concentration of dipyrone in each dilution and the intensity of $\mathrm{PC} 1$ in each concentration (in triplicate). This linear equation $(y=0.0003322 x-0.01034)$, a regression curve of $\mathrm{PC} 1$ versus concentration, was then used to estimate the concentration in the same samples (Figure 4(b)). An
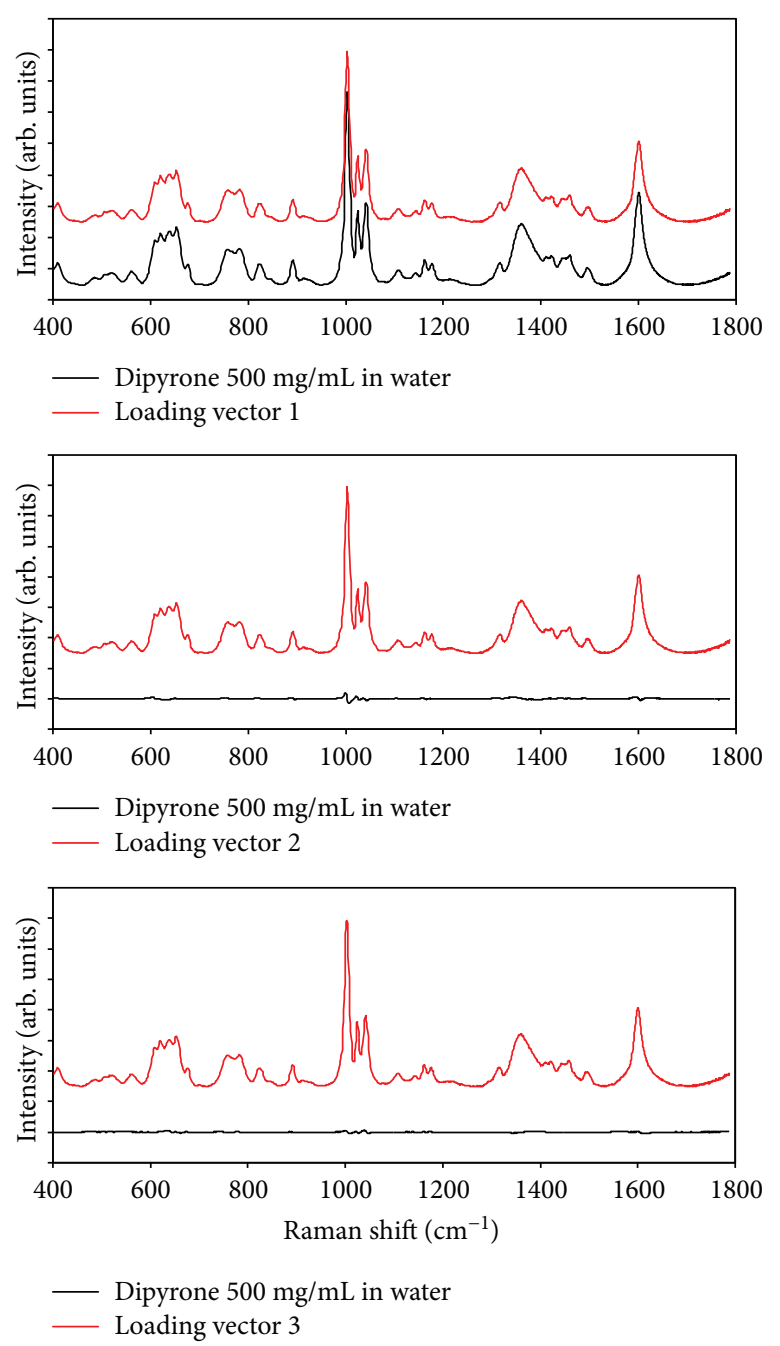

FIgURE 3: Raman spectra of a solution of $500 \mathrm{mg} / \mathrm{mL}$ of dipyrone diluted in distilled water $(100 \%$ dilution) and the reference commercial solution of dipyrone $500 \mathrm{mg} / \mathrm{mL}$. Table 1 presents the labeled peaks in the reference solution that are related to dipyrone and their assignments. Inner plot: metamizole chemical formula $\{[(1,5$-dimethyl-3-oxo-2-phenylpyrazol-4-yl)-methylamino] methanesulfonic acid sodium salt\}.

excellent agreement of the data points can be seen with the zero-error diagonal, revealing an excellent model fit.

By applying the PCR model in the Raman spectra of triplicate samples, it is possible to calculate the predicted concentration and provide a root mean squared error (RMSE) of prediction [41], to which the model is subject. The RMSE obtained in this correlation curve was $6.5 \mathrm{mg} / \mathrm{mL}(1.3 \%)$. The resulting prediction error is below that preconized by the Brazilian Sanitary Surveillance Agency [8], which is 5\%.

3.2. Figures of Merit for the PCR Model. The results of ANVISA figures of merit for the ANVISA class I parameters are presented.

(i) Interval: The range of 400 to $600 \mathrm{mg} / \mathrm{mL}$ (80 to $120 \%$ ) was established. 
TABle 1: Assignments of the main Raman bands of dipyrone found in Figure 3 based on the literature of vibrational spectroscopy.

\begin{tabular}{|c|c|}
\hline Peak position $\left(\mathrm{cm}^{-1}\right)$ & Assignment \\
\hline 563 & $\mathrm{SO}_{2}$ scissor deformation (sulfone) [37] \\
\hline $600-700$ & Ring in-plane and out-of-plane deformations (phenyl group) \\
\hline $740-790$ & C-H out-of-plane bending (phenyl group) [38] \\
\hline 1003 & $\begin{array}{c}\mathrm{C}=\mathrm{C} \text { stretching and angular } \mathrm{CCC} \text { in-plane ring deformation (symmetric stretching-"breathing" of the } \\
\text { phenyl ring) }[37,39]\end{array}$ \\
\hline 1100 & $\mathrm{SO}_{2}$ symmetric stretch (sulfone) [37] \\
\hline 1359 & $\mathrm{SO}_{2}$ antisymmetric stretch (sulfone) [37] \\
\hline 1459 & $\mathrm{C}=\mathrm{C}$ ring stretching (degenerate vibrations pair of the phenyl ring, associated to the symmetric out-of-phase and \\
\hline 1495 & antisymmetric stretching of the two triatomic systems); $\mathrm{N}-\mathrm{CH}_{3}$ deformation (amine) $[38,40]$ \\
\hline 1601 & $\mathrm{C}=\mathrm{O}$ and $\mathrm{C}=\mathrm{C}$ stretching (phenyl group); $\mathrm{HCH}$ deformation $\left(\mathrm{CH}_{3}\right.$ an $\mathrm{CH}_{2}$ groups) [39] \\
\hline
\end{tabular}

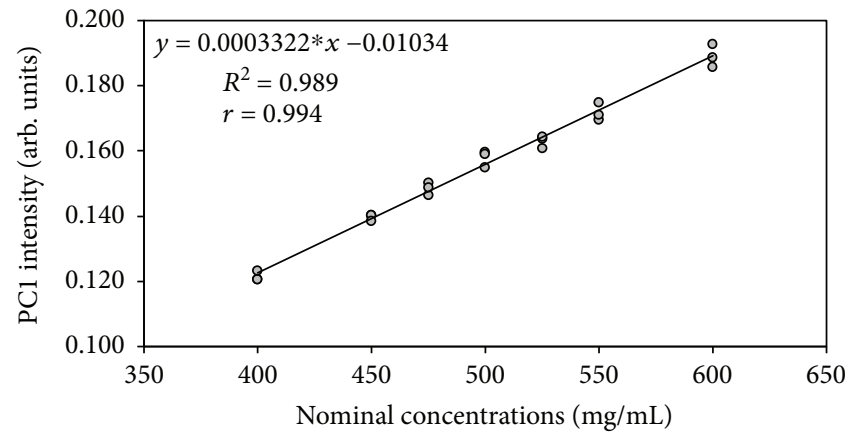

(a)

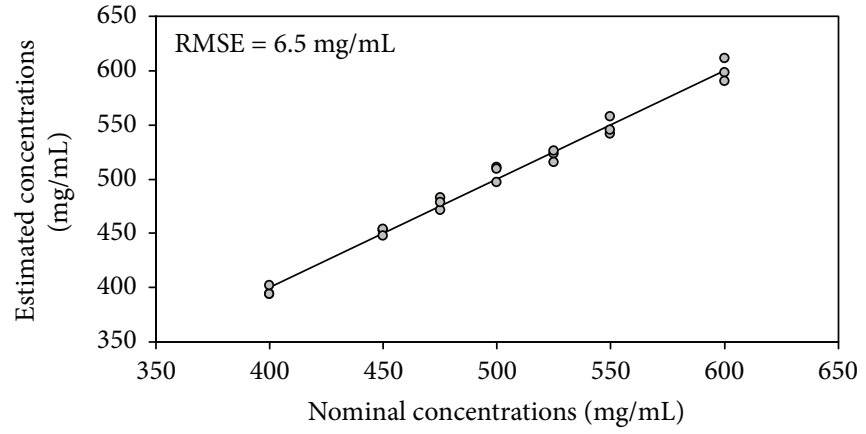

(b)

Figure 4: (a) Plot of the nominal concentrations of the seven dilutions of dipyrone, in the range from 400 to $600 \mathrm{mg} / \mathrm{mL}$ ( 80 to $120 \%$ ) versus the intensities of PC1, showing high correlation of the PCR model $(r=0.994)$. (b) Plot of the model-estimated concentrations versus the nominal concentrations and estimated $\mathrm{RMSE}=6.5 \mathrm{mg} / \mathrm{mL}(1.3 \%)$.

(ii) Specificity/selectivity: The largest relative error of dipyrone in full excipient compared to dipyrone in water was $3.3 \%$, showing that the technique allows the detection of the major component of the sample in different excipients (Table 2).

(iii) Linearity: The concentrations of five solutions were determined at different concentrations of the drug in water $(400,450,475,500,525,550$, and $600 \mathrm{mg} / \mathrm{mL}$ ) and with the concentrations of the real dilutions. These data are shown in Figure 4(a). The curve obtained to verify the linearity was $y=0.0003322 x-0.01034(y=m x+b$, where $m$ is the slope and $b$ is the intercept of the line with the vertical axis). The linear regression coefficient obtained was $r=0.994$, indicating a high correlation between the dilution effectively performed and the predicted concentration.

(iv) Precision (repeatability): Three concentrations (low, intermediate, and high) were used with three replicate samples of the following concentrations: 400, 500 , and $600 \mathrm{mg} / \mathrm{mL}$. RSD was calculated for each concentration obtained by the PCR model in comparison with the real dilution values. ANVISA criteria accept values of precision (RSD) $\leq 5 \%$. Table 3 presents the data used to calculate the RSD for the solutions of low, intermediate, and high concentrations $(400,500$, and $600 \mathrm{mg} / \mathrm{mL})$. It was found that all three concentrations presented an RSD below the maximum limit specified by ANVISA, ranging from 0.95 to $1.76 \%$.

(v) Accuracy: this was calculated by using the predicted concentrations of 400,500 , and $600 \mathrm{mg} / \mathrm{mL}$, in triplicate, being determined by means of the relative deviation (RD) and recovery, as shown in Table 3. The acceptance criteria established were $\mathrm{RD} \leq 2 \%$, and satisfactory recovery values are found within the range from 98 and $102 \%$ [8]. The solutions showed a relative deviation lower than $1.5 \%$, and the recovery showed values ranging from $99.2 \%$ to $101.2 \%$, being within the limits of acceptance.

(vi) Robustness: Due to the lack of evaluation of the influence of hydrogen potential and temperature on the results of the present study, the robustness of the method was not estimated. 
TABle 2: Predicted concentrations of dipyrone $500 \mathrm{mg} / \mathrm{mL}$ in water and in the reference commercial pharmaceutical preparation using the PCR model and the relative errors used to calculate the selectivity.

\begin{tabular}{lccc}
\hline Sample & $\begin{array}{c}\text { Predicted } \\
\text { concentration of } \\
\text { dipyrone diluted in } \\
\text { water }(\mathrm{mg} / \mathrm{mL})\end{array}$ & $\begin{array}{c}\text { Predicted } \\
\text { concentration } \\
\text { of dipyrone in the } \\
\text { reference commercial } \\
\text { pharmaceutical } \\
\text { preparation }(\mathrm{mg} / \mathrm{mL})\end{array}$ & $\begin{array}{c}\text { Relative } \\
\text { error }(\%)\end{array}$ \\
\hline 1 & 511 & 505 & $1.2 \%$ \\
2 & 497 & 485 & $2.4 \%$ \\
3 & 509 & 492 & $3.3 \%$ \\
\hline
\end{tabular}

TABLE 3: Solutions of different concentrations in triplicate $(400,500$, and $600 \mathrm{mg} / \mathrm{mL}$ ) used to calculate the precision (RSD) and accuracy (AC).

\begin{tabular}{lcccccc}
\hline $\begin{array}{l}\text { Real } \\
\begin{array}{l}\text { concentration } \\
(\mathrm{mg} / \mathrm{mL})\end{array}\end{array}$ & $\begin{array}{c}\mathrm{VO} / \mathrm{PCR} \\
(\mathrm{mg} / \mathrm{mL})\end{array}$ & $\begin{array}{c}\mathrm{DAC} \\
(\mathrm{mg} / \mathrm{mL})\end{array}$ & $\begin{array}{c}\mathrm{SD} \\
(\mathrm{mg} / \mathrm{mL})\end{array}$ & $\begin{array}{c}\mathrm{RSD} \\
(\%)\end{array}$ & $\begin{array}{c}\mathrm{RD} \\
(\%)\end{array}$ & $\begin{array}{c}\mathrm{AC} \\
(\%)\end{array}$ \\
\hline \multirow{2}{*}{400} & 395 & & & & & \\
& 394 & 397 & 3.81 & 0.95 & 0.75 & 99.2 \\
& 401 & & & & & \\
500 & 511 & & & & & \\
& 497 & 506 & 7.58 & 1.49 & 1.2 & 101.2 \\
& 509 & & & & & \\
600 & 611 & & & & & \\
& 598 & 600 & 10.61 & 1.76 & 0 & 100.0 \\
& 590 & & & & & \\
\hline
\end{tabular}

VO: value obtained in the assay test of RS/PCR; RS/PCR: Raman spectroscopy combined with chemometric method PCR; DAC: determined average concentration; $\mathrm{RD}$ : relative deviation.

3.3. Quantitative Model Applied to the Commercial Formulations. The PCR model has been applied to estimate the concentration of API in the commercial products. The PCs of each medicine sample spectrum were estimated by least-square fitting the first three LVs and the medicine spectra, fitting the coefficient of PCs. Since PC1 was used for the PCR model applied to the samples of dipyrone diluted in water, the concentration of dipyrone in each medicine sample was obtained by estimating $x$ in the linear equation of Figure 4(a) $[x=(y+0.01034) / 0.003322]$. The predicted concentrations of the 10 samples of commercial medicines through the PCR model were correlated to the concentrations presented in the label (Figure 5), and all samples fell below the $\pm 5 \%$ error line, indicating excellent results in determining the API concentration.

\section{Discussion}

The pharmaceutical industry is seeking methods to better track drugs throughout the production process, since most of the high-performance techniques currently used require sample preparation using solvents, are destructive and time-

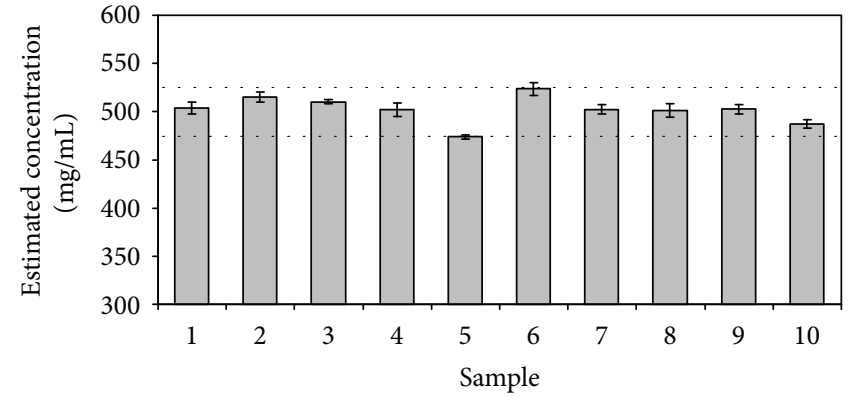

FIgURE 5: Estimated concentration values using the PCR model applied to the Raman spectra of the commercial solutions containing dipyrone $500 \mathrm{mg} / \mathrm{mL}$. The error bars are based on the triplicate spectra. The $\pm 5 \%$ precision limit of ANVISA is shown.

consuming, and mostly need expensive inputs $[8,16,42]$. The use of PAT in the pharmaceutical industry and in biotechnological processes led to advances regarding the ability to analyze/monitor key process and quality attributes in the biotech industry, to utilize the collected data for the subsequent control of the process, in order to achieve the optimum yield and product quality [43]. The literature indicates that Raman spectroscopy, combined with chemometric methods such as PLSR and PCR, would meet the challenges for a reliable quantitative determination of API in pharmaceutical drugs [44, 45].

The present study was based on the spectral model to assay dipyrone in commercial medicines, in order to evaluate this optical technique as a method for on-line and at-line analysis, in accordance with the PAT requirements for an industrial analysis system. PCR has been employed for quantitative measurements in chemical analysis when applied to spectroscopic methods $[24,25]$. In complex and multivariate systems, such as API in medicines and formulations, the classical univariate regression method may become unreliable because of possible band interactions with the solvents and excipients and band superimpositions. The PCR models all variables that are correlated to the concentration of the API, and thus, the calibration can be performed efficiently, even in the presence of interferences using the whole of the spectral information. With the PCR model, new samples can be modeled at any time. The PCR model showed a smaller prediction error when using the first principal component score. In this way, it was verified that only one spectral variation was found in the samples, which is related to changes in the dipyrone concentration.

Since the intensity of Raman peaks is directly proportional to the concentration of the components on the scattering sample [46], Raman could be used to develop linear models for the evaluation of a medicine's composition. Since the intensity of a Raman band is dependent on the changes in the molecule's polarizability presented in the sample, this has a relevant consequence in investigating pharmaceutical materials because active ingredients are organic molecules that contain covalent bonds and usually aromatic conjugated systems, which show strong Raman transitions, in contrast to the excipients in most formulations, which are generally weaker Raman scatterers due to a lack of $\mathrm{C}$ bonds. 
In this work, PCR was validated by evaluating the ANVISA RE no. 899/2003 figures of merit such as specificity, linearity, precision, and accuracy in the same way as Orkoula et al. [47], Rossignoli et al. [48], and Borio et al. [49]. The PCR model showed excellent linearity with a correlation coefficient $r=0.994$, high precision, and RSD as low as $1.76 \%$. Corroborating the method validation employed by Orkoula et al. [47], commercial samples of the reference drug containing $500 \mathrm{mg} / \mathrm{mL}$ of dipyrone were used to validate the model. These samples showed very low errors, not exceeding $2.5 \%$, suggesting that dispersive Raman spectroscopy using an optical probe can detect the amount of API present in the commercial samples, and all samples conformed, which is different to the study of Knappmann and Melo [32].

Although dipyrone has been used for more than 90 years, research about quality control, harm, and health risk of this active ingredient is scarce [50]. Dipyrone concentration has been evaluated in several commercial drugs containing dipyrone by FT-Raman spectroscopy [39]; the results showed agreement with the percentages of dipyrone present in tablets. In Brazil, the quality control of medicines is restricted to certain government laboratories [39]. Despite this, Pereira et al. [51] performed the spectrophotometric determination of dipyrone in commercial samples by flow injection triiodide generation, with sixty results obtained per hour. The results found were within the 3\% error range at the $95 \%$ confidence level. The quality control of distinct brands of dipyrone in oral solutions, marketed in Brazilian drugstores, was also determined by Knappmann and Melo [32], and the results indicated that the quality control of similar medicines needs to be improved, since similar drugs were the ones that presented the most deviations of quality, being disapproved in the study trial.

Dispersive Raman spectroscopy has been considered the most suitable technique to measure API in different pharmaceutical preparations, with advantages such as the ability to obtain a spectrum with a high signal-to-noise ratio using a low-power near-infrared laser with reduced exposure time. In addition, the entire spectrum is obtained at once through a multichannel detector and imaging spectrograph.

According to the PAT guide, the gains in processes quality, safety, and/or efficiency can be increased depending on the product; these changes come from factors such as the prevention of rejections, the disposal and reprocessing of products, and the possibility of carrying out analysis in real time with reduced or no sample preparation $[5,52]$, leading to a reduction of human error and increasing the safety of the operator, with no risk to the environment $[9,10,53]$. The PCR model could address most of these factors, because each sample can be irradiated rapidly and examined directly with the use of an optical probe, without prior preparation, to obtain a single spectrum, which minimizes human error. Furthermore, the use of Raman spectroscopy as an analytical technique offers the possibility of measurements in real time at-line, avoiding the storage time required until the medicine's stock release, preventing possible rejections/disposal by furnishing actual concentration values, and even measuring the concentration through containers by standard Raman and offset spatially Raman spectroscopy [54-56].

\section{Conclusions}

This study demonstrated the potential of dispersive Raman spectroscopy to attend ANVISA figures of merit for class I validation (specificity, linearity, range, precision, and accuracy) for evaluation of the concentration of dipyrone in commercial solutions. An effectiveness of the quantitative method was found, with high linearity, specificity of $3.3 \%$, precision $<2 \%$, and relative deviation (accuracy) $<1.5 \%$, with recovery values in the range of $99.2 \%$ to $101.2 \%$ and RMSE of $6.5 \mathrm{mg} / \mathrm{mL}$ (1.3\%) for quantifying dipyrone diluted in water. For the quantification of API in the commercial formulation, the model showed concentration values closer to $500 \mathrm{mg} / \mathrm{mL}$, with an error of less than $2.5 \%$ compared to the labeled concentration. It has been demonstrated that the PCR model could support the industry's applications of monitoring and quality control, rapidly and without any sample preparation, attending the PAT requirements of the pharmaceutical industry for quality assurance of the final product due to the security and agility provided.

\section{Conflicts of Interest}

The authors declare that there is no conflict of interests regarding the publication of this article.

\section{Acknowledgments}

Landulfo Silveira Jr. thanks FAPESP (São Paulo Research Foundation) for the grant for the Raman spectrometer (Grant no. 2009/01788-5) and CNPq (National Council for Scientific and Technological Development) (Grant no. 305680/2014-5) for the productivity fellowship. Letícia Parada Moreira and Bárbara Faria Lourenço would like to acknowledge CAPES (Coordenação de Aperfeiçoamento de Pessoal de Nível Superior) for the scholarships.

\section{References}

[1] J. Konijnenbelt-Peters, C. Van der Heijden, C. Ekhart, J. Bos, J. Bruhn, and C. Kramers, "Metamizole (dpyrone) as an alternative agent in postoperative analgesia in patients with contraindications for nonsteroidal anti-inflammatory drugs," Pain Practice, vol. 17, no. 3, pp. 402-408, 2017.

[2] A. Jasiecka, T. Maślanka, and J. J. Jaroszewski, "Pharmacological characteristics of metamizole," Polish Journal of Veterinary Sciences, vol. 17, no. 1, pp. 207-214, 2014.

[3] L. Lachman, H. A. Lieberman, and J. L. Kanig, The Theory and Practice of Industrial Pharmacy, Lea \& Febiger, Philadelphia, PA, USA, 3rd edition, 1986.

[4] Brazil, Brazilian Pharmacopoeia, Brazilian Sanitary Surveillance Agency, Brazil, 5th edition, 2010.

[5] D. C. Hinz, "Process analytical technologies in the pharmaceutical industry: the FDA's PAT initiative," Analytical and Bioanalytical Chemistry, vol. 384, no. 5, pp. 1036-1042, 2006.

[6] F. Adar, R. Geiger, and J. Noonan, "Raman spectroscopy for process/quality control," Applied Spectroscopy Reviews, vol. 32, no. 1-2, pp. 45-101, 1997. 
[7] G. J. Fini, "Applications of Raman spectroscopy to pharmacy," Journal of Raman Spectroscopy, vol. 35, no. 5, pp. 335-337, 2004.

[8] Brazil, Resolution RE No. 899/2003, "Ministry of Health. Brazilian Sanitary Surveillance Agency (ANVISA), 2003," October 2017, https://anvisa.gov.br/legis/resol/2003/re/899_ 03re.htm.

[9] Food and Drug Administration, International Conference on Harmonization, "Guideline on Validation of Analytical Procedures: Definitions and Terminology; Availability," Federal Register, vol. 60, no. 40, pp. 11260-11262, 1995.

[10] Food and Drug Administration, International Conference on Harmonization, "Guideline on the Validation of Analytical Procedures: Methodology; Availability," Federal Register, vol. 62, no. 96, pp. 27463-27467, 1997.

[11] A. W. Newman and S. R. Byrn, "Solid-state analysis of the active pharmaceutical ingredient in drug products," Drug Discovery Today, vol. 8, no. 19, pp. 898-905, 2003.

[12] M. Blanco, M. Bautista, and M. Alcalà, "API determination by NIR spectroscopy across pharmaceutical production process," AAPS PharmSciTech, vol. 9, no. 4, pp. 1130-1135, 2008.

[13] O. Kolomiets, U. Hoffmann, P. Geladi, and H. W. Siesler, "Quantitative determination of pharmaceutical drug formulations by near-infrared spectroscopic imaging," Applied Spectroscopy, vol. 62, no. 11, pp. 1200-1208, 2008.

[14] C. J. Strachan, T. Rades, K. C. Gordon, and J. Rantanen, "Raman spectroscopy for quantitative analysis of pharmaceutical solids," Journal of Pharmacy and Pharmacology, vol. 59, no. 2, pp. 179-192, 2007.

[15] T. Vankeirsbilck, A. Vercauteren, W. Baeyens et al., "Applications of Raman spectroscopy in pharmaceutical analysis," TrAC Trends in Analytical Chemistry, vol. 21, no. 12, pp. 869-9877, 2002.

[16] C. Eliasson, N. A. Macleod, L. C. Jayes et al., "Non-invasive quantitative assessment of the content of pharmaceutical capsules using transmission Raman spectroscopy," Journal of Pharmaceutical and Biomedical Analysis, vol. 47, no. 2, pp. 221-229, 2008.

[17] B. B. Koleva, T. M. Kolev, D. L. Tsalev, and M. Spiteller, "Determination of phenacetin and salophen analgesics in solid binary mixtures with caffeine by infrared linear dichroic and Raman spectroscopy," Journal of Pharmaceutical and Biomedical Analysis, vol. 46, no. 2, pp. 267-273, 2008.

[18] E. B. Hanlon, R. Manoharan, T. W. Koo et al., "Prospects for in vivo Raman spectroscopy," Physics in Medicine \& Biology, vol. 45, no. 2, pp. R1-R59, 2000.

[19] M. S. Hwang, S. Cho, H. Chung, and Y. A. Woo, "Nondestructive determination of the ambroxol content in tablets by Raman spectroscopy," Journal of Pharmaceutical and Biomedical Analysis, vol. 38, no. 2, pp. 210-215, 2005.

[20] P. R. Mobley, B. R. Kowalski, J. J. Workman, and R. Bro, "Review of chemometrics applied to spectroscopy: 1985-95, Part 2," Applied Spectroscopy Reviews, vol. 31, no. 4, pp. 347368, 1996.

[21] M. M. C. Ferreira, A. M. Antunes, M. S. Melgo, and P. L. O. Volpe, "Chemometrics I: multivariate calibration, a tutorial," Química Nova, vol. 22, no. 5, pp. 724-731, 1999.

[22] M. M. Sena and R. J. Poppi, "N-way PLS applied to simultaneous spectrophotometric determination of acetylsalicylic acid, paracetamol and caffeine," Journal of Pharmaceutical and Biomedical Analysis, vol. 34, no. 1, pp. 27-34, 2004.
[23] P. Geladi and B. R. Kowalski, "Partial least-squares regression: a tutorial," Analytica Chimica Acta, vol. 185, pp. 1-17, 1986.

[24] S. Boneh and G. R. Mendieta, "Regression modeling using principal components," in 4th Annual Conference on Applied Statistics in Agriculture, Manhattan, KS, USA, September 19922017, http://newprairiepress.org/agstatconference/1992/ proceedings $/ 20$.

[25] "The Mathworks Documentation, Partial Least Squares Regression and Principal Components Regression," September 2017, https://www.mathworks.com/help/stats/examples/ partial-least-squares-regression-and-principal-componentsregression.html.

[26] R. Szostak and S. Mazurek, "Quantitative determination of acetylsalicylic acid and acetaminophen in tablets by FTRaman spectroscopy," Analyst, vol. 127, no. 1, pp. 144-148, 2002.

[27] M. C. Ferraro, P. M. Castellano, and T. S. Kaufman, "Chemometric determination of amiloride hydrochloride, atenolol, hydrochlorothiazide and timolol maleate in synthetic mixtures and pharmaceutical formulations," Journal of Pharmaceutical and Biomedical Analysis, vol. 34, no. 2, pp. 305-314, 2004.

[28] A. P. do Nascimento, M. G. Trevisan, E. R. M. KedorHackmann, and R. J. Poppi, "Simultaneous determination of dipyrone and papaverine in pharmaceutical formulation using PLS regression and UV spectrophotometry," Analytical Letters, vol. 40, no. 5, pp. 975-986, 2007.

[29] S. Mazurek and R. Szostak, "Quantitative determination of diclofenac sodium in solid dosage forms by FT-Raman spectroscopy," Journal of Pharmaceutical and Biomedical Analysis, vol. 48, no. 3, pp. 814-821, 2008.

[30] W. F. C. Rocha, A. L. Rosa, J. A. Martins, and R. J. Poppi, "Determination and validation of nimesulide in pharmaceutical formulation by near infrared spectroscopy," Journal of the Brazilian Chemical Society, vol. 21, no. 10, pp. 1929-2010, 2010.

[31] M. Edinger, D. Bar-Shalom, J. Rantanen, and N. Genina, "Visualization and non-destructive quantification of inkjetprinted pharmaceuticals on different substrates using Raman spectroscopy and Raman chemical imaging," Pharmaceutical Research, vol. 34, no. 5, pp. 1023-1036, 2017.

[32] A. L. Knappmann and E. B. D. Melo, "Quality of over-thecounter medicines: a study with dipyrone brands commercialized in a drugstore in Cascavel city (Paraná, Brazil)," Ciência \& Saúde Coletiva, vol. 15, suppl 3, pp. 3467-3476, 2010.

[33] F. Arellano and J. A. Sacristán, "Metamizole: reassessment of its therapeutic role," European Journal of Clinical Pharmacology, vol. 38, no. 6, pp. 617-619, 1991.

[34] B. Baro-Oz, M. Clementi, E. Di Giantonio et al., "Metamizol (dipyrone, optalgin) in pregnancy, is it safe? A prospective comparative study," European Journal of Obstetrics \& Gynecology and Reproductive Biology, vol. 119, no. 2, pp. 176-179, 2005.

[35] C. A. Lieber and A. Mahadevan-Jansen, "Automated method for subtraction of fluorescence from biological Raman spectra," Applied Spectroscopy, vol. 57, no. 11, pp. 1363-1367, 2003.

[36] A. C. Olivieri, N. M. Faber, J. Ferré, R. Boqué, J. H. Kalivas, and H. Mark, "Uncertainty estimation and figures of merit for multivariate calibration (IUPAC Technical Report)," Pure and Applied Chemistry, vol. 78, no. 3, pp. 633-661, 2006. 
[37] N. Colthup, L. Daly, and S. Wiberley, Introduction to Infrared and Raman Spectroscopy, Academic Press, London, 1990.

[38] D. W. Mayo, F. A. Miller, and R. W. Hannah, Course Notes on the Interpretation of Infrared and Raman Spectra, John Wiley \& Sons, Hoboken, NJ, USA, 2004.

[39] A. O. Izolani, M. T. de Moraes, S. Téllez, and C. A. Claudio, "Fourier transform Raman spectroscopy of drugs: quantitative analysis of 1-phenyl-2,3-dimethyl-5-pyrazolone-4-methylaminomethane sodium sulfonate: (dipyrone)," Journal of Raman Spectroscopy, vol. 34, no. 10, pp. 837-843, 2003.

[40] D. Lin-Vien, N. B. Colthup, W. G. Fateley, and J. G. Grasselli, The Handbook of Infrared and Raman Characteristic Frequencies of Organic Molecules, Academic Press, San Diego, CA, USA, 1991.

[41] H. Martens and M. Martens, Multivariate Analysis of Quality: An Introduction, John Wiley \& Sons, West Sussex, 2001.

[42] B. A. Olsen, "Developing and using analytical methods to achieve quality by design and efficiency in drug development," Pharmaceutical Technology, vol. 1, pp. S14-S25, 2005.

[43] A. S. Rathore, R. Bhambure, and V. Ghare, "Process analytical technology (PAT) for biopharmaceutical products," Analytical Bioanalytical Chemistry, vol. 398, no. 1, pp. 137-154, 2010.

[44] M. Kim, H. Chung, Y. Woo, and M. S. Kemper, "A new noninvasive, quantitative Raman technique for the determination of an active ingredient in pharmaceutical liquids by direct measurement through a plastic bottle," Analytica Chimica Acta, vol. 587, no. 2, pp. 200-207, 2007.

[45] E. Katainen, M. Elomaa, U. M. Laakkonen et al., "Quantification of the amphetamine content in seized street samples by Raman spectroscopy," Journal of Forensic Sciences, vol. 52, no. 1, pp. 88-92, 2007.

[46] European Pharmacopoeia 8.7, Chapter 2.2.48: Raman spectroscopy, European Directorate for the Quality of Medicines \& HealthCare, Council of Europe, Strasbourg, 2016, p. 8.7-5464.

[47] M. G. Orkoula, C. G. Kontoyannis, C. K. Markopoulou, and J. E. Koundourellis, "Quantitative analysis of liquid formulations using FT-Raman spectroscopy and HPLC: The case of diphenhydramine hydrochloride in Benadryl," Journal of Pharmaceutical and Biomedical Analysis, vol. 41, no. 4, pp. 1406-1411, 2006.

[48] P. Rossignoli, R. Pontarolo, C. J. Correr, G. Cordeiro, and P. Peralta-Zamora, "Multivariate spectroscopic determination of glucosamine and chondroitin in pharmaceutical formulations," Química Nova, vol. 31, no. 6, pp. 1285-1289, 2008.

[49] V. G. Borio, R. Vinha, R. A. Nicolau, H. P. M. de Oliveira, C. J. de Lima, and L. Silveira, "Quantitative evaluation of acetaminophen in oral solutions by dispersive Raman spectroscopy for quality control," Spectroscopy: An International Journal, vol. 27, no. 4, pp. 215-228, 2012.

[50] T. Kötter, B. R. da Costa, M. Fässler et al., "Metamizole-associated adverse events: a systematic review and meta-analysis," PLoS One, vol. 10, no. 4, article e0122918, 2015.

[51] A. V. Pereira, L. Penckowski, M. Vosgerau, M. F. Sassá, and O. Fatibello Filho, "Spectrophotometric determination of metamizol in pharmaceutical formulations by flow injection triiodide generation,” Química Nova, vol. 25, no. 4, pp. 553-557, 2002.

[52] Food and Drug Administration, "Guidance for industry PAT a framework for innovative pharmaceutical development, manufacturing, and quality assurance, 2004," October 2017 https://www.fda.gov/downloads/drugs/guidances/ucm070305. pdf.
[53] R. P. Cogdill, C. A. Anderson, M. Delgado et al., "Process analytical technology case study: part II. Development and validation of quantitative near-infrared calibrations in support of a process analytical technology application for real-time release," AAPS PharmSciTech, vol. 6, no. 2, pp. E273-E283, 2005.

[54] I. Malik, M. Poonacha, J. Moses, and R. A. Lodder, "Multispectral imaging of tablets in blister packaging," AAPS PharmSciTech, vol. 2, no. 2, pp. 38-44, 2001.

[55] P. De Peinder, M. J. Vredenbregt, T. Visser, and D. J. De Kaste, "Detection of Lipitor ${ }^{\circledR}$ counterfeits: a comparison of NIR and Raman spectroscopy in combination with chemometrics," Journal of Pharmaceutical and Biomedical Analysis, vol. 47, no. 4-5, pp. 688-694, 2008.

[56] K. Dégardin, A. Guillemain, and Y. Roggo, "Comprehensive study of a handheld Raman spectrometer for the analysis of counterfeits of solid-dosage form medicines," Journal of Spectroscopy, vol. 2017, Article ID 3154035, 13 pages, 2017. 

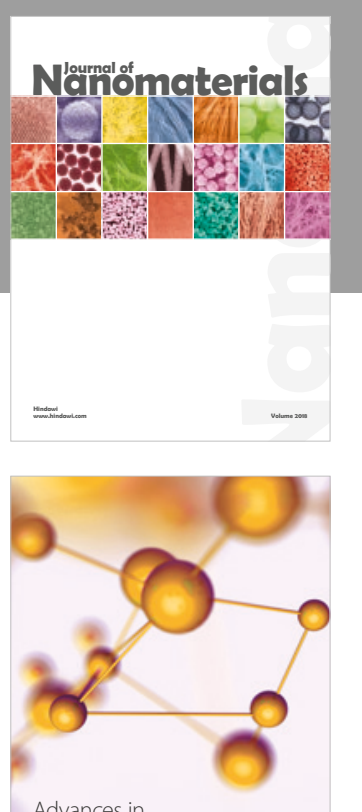

Physical Chemistry
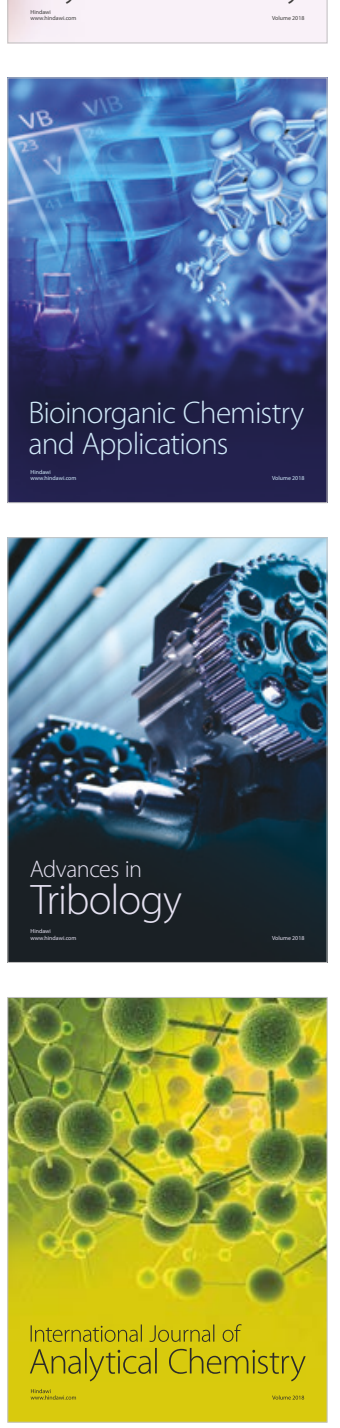

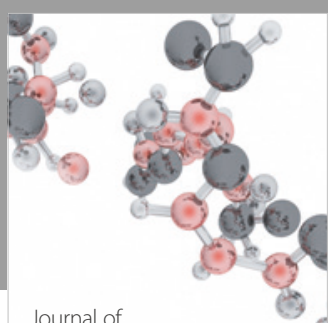

Analytical Methods

in Chemistry

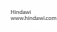

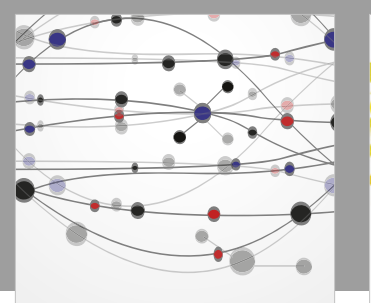

The Scientific World Journal

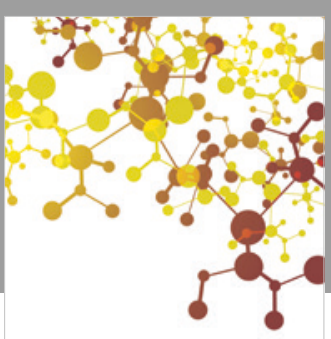

Journal of

Applied Chemistry
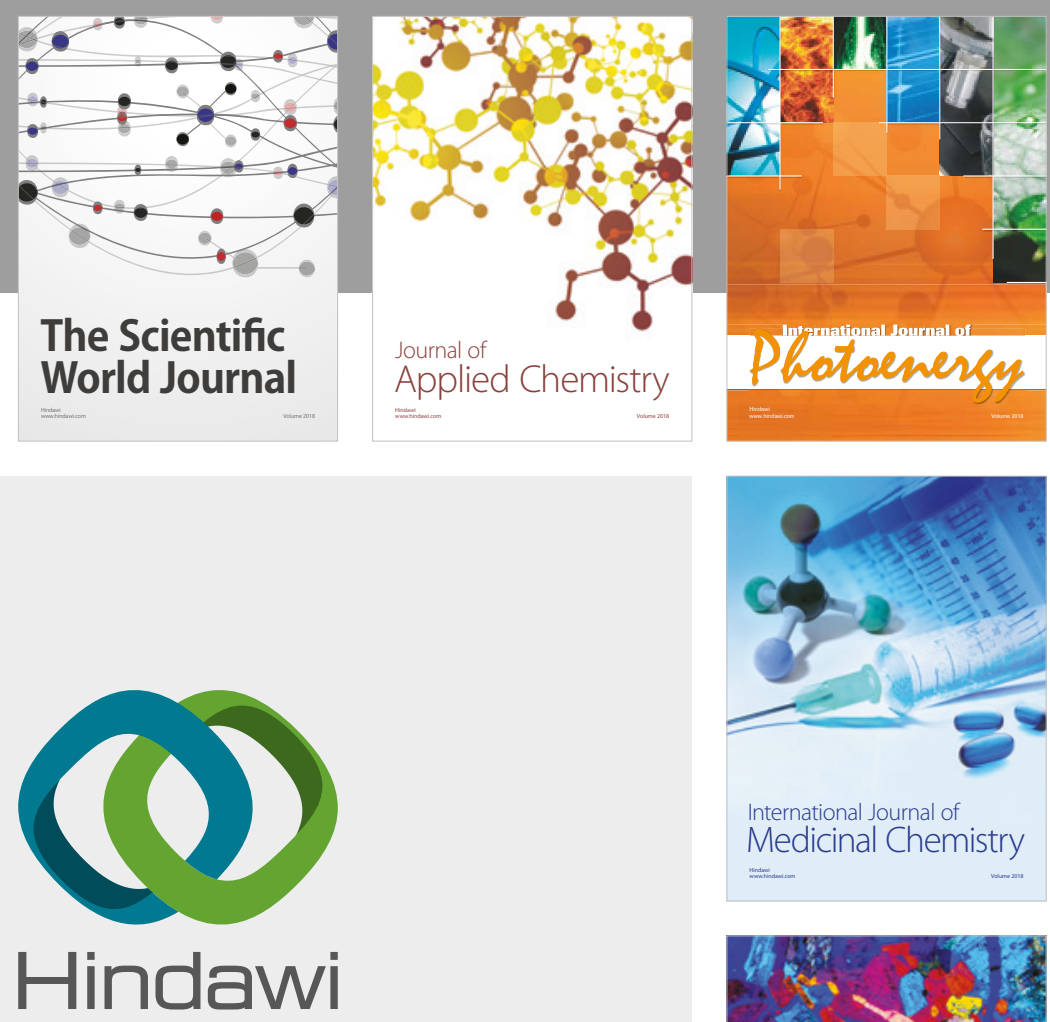

Submit your manuscripts at

www.hindawi.com
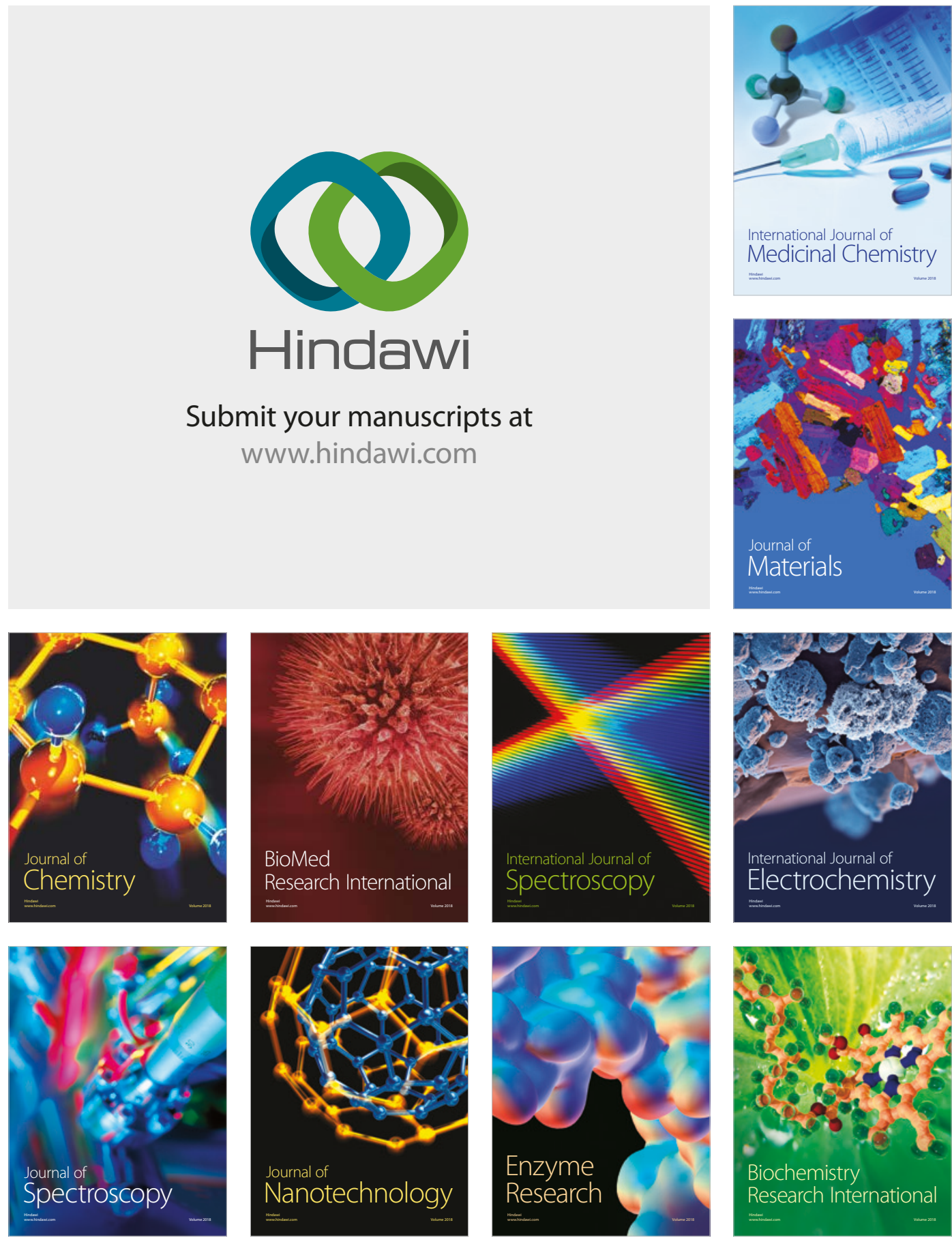
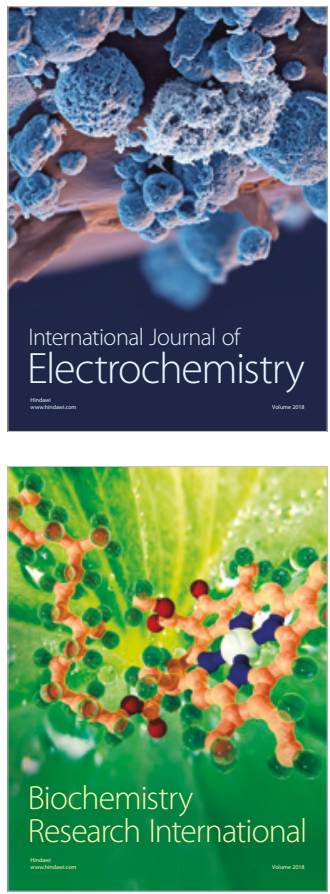